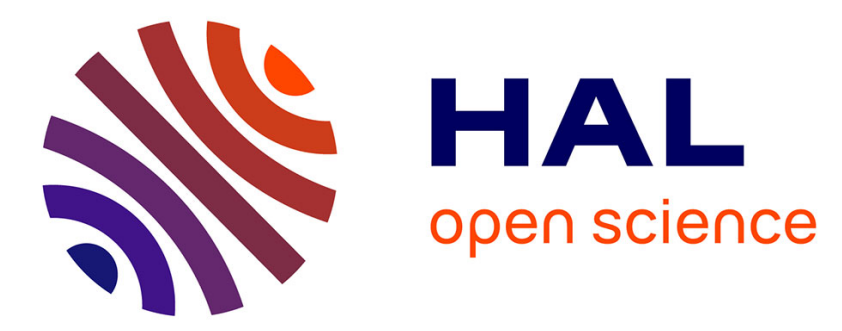

\title{
A reference accelerator scheme for ADS applications
}

\author{
J.-L. Biarrotte, S. Bousson, T. Junquera, A.C. Mueller, A. Olivier
}

\section{To cite this version:}

J.-L. Biarrotte, S. Bousson, T. Junquera, A.C. Mueller, A. Olivier. A reference accelerator scheme for ADS applications. 7th International Conference on Accelerator Applications - AccApp05, Aug 2005, Venice, Italy. pp.656-661, 10.1016/j.nima.2006.02.127 . in2p3-00106863

\section{HAL Id: in2p3-00106863 https://hal.in2p3.fr/in2p3-00106863}

Submitted on 16 Oct 2006

HAL is a multi-disciplinary open access archive for the deposit and dissemination of scientific research documents, whether they are published or not. The documents may come from teaching and research institutions in France or abroad, or from public or private research centers.
L'archive ouverte pluridisciplinaire HAL, est destinée au dépôt et à la diffusion de documents scientifiques de niveau recherche, publiés ou non, émanant des établissements d'enseignement et de recherche français ou étrangers, des laboratoires publics ou privés. 


\title{
A reference accelerator scheme for ADS applications
}

\author{
Jean-Luc Biarrotte $^{\mathrm{a}, *}$, Sébastien Bousson $^{\mathrm{a}}$, Tomas Junquera ${ }^{\mathrm{a}}$, Alex C. Mueller ${ }^{\mathrm{a}}$, \\ Aurélia Olivier ${ }^{\mathrm{a}}$ \\ ${ }^{a}$ CNRS/IN2P3, Institut de Physique Nucléaire, 91406 Orsay, France
}

Elsevier use only: Received date here; revised date here; accepted date here

\begin{abstract}
Accelerator Driven Systems (ADS) for transmutation of nuclear waste typically require $350 \mathrm{MeV}-1 \mathrm{GeV}$ accelerators delivering proton fluxes of $5-10 \mathrm{~mA}$ for demonstrators, and $20-50 \mathrm{~mA}$ for large industrial systems. Thus, such machines belong to the category of the so-called HPPA (High-Power Proton Accelerators), with multi-megawatt beam power. HPPA are presently developed and constructed at great pace for their broad utility in fundamental or applied science. Compared to other HPPA, many features and requirements are similar for the ADS driver. However, there is a need for exceptional reliability: because of the induced thermal stress to the subcritical core, the number of unwanted "beam-trips" should not exceed a few per year, a requirement that is several orders of magnitude above usual performance. Consecutive to the work of the European Technical Working Group (ETWG) on ADS, the Preliminary Design Study of an Experimental ADS (PDSXADS) was launched in 2001 as a $5^{\text {th }}$ Framework Program EC project ${ }^{\dagger}$. A special Working Package (WP3) was dedicated to the accelerator design, taking especially into account that the issue of "beam-trips" could be a potential "show-stopper" for ADS technology in general. A reference solution, based on a linear superconducting accelerator with its associated doubly achromatic beam line, has been worked out to some detail. For high reliability, the proposed design is intrinsically fault tolerant, relying on highly modular "de-rated" components associated to a fast digital feedback system. The proposed solution also appears to be robust concerning operational aspects like maintenance and radioprotection. A roadmap for construction as well as the required consolidated budget was elaborated. A program for the remaining $R \& D$, focused on experimental reliability demonstration of prototypical components has been elaborated. This R\&D will be performed in the $6^{\text {th }}$ Framework Program EC project EUROTRANS ${ }^{\ddagger}$, which presently is just starting. (c) 2001 Elsevier Science. All rights reserved
\end{abstract}

\footnotetext{
${ }^{*}$ Corresponding author. Tel.: +33-1-69-15-79-30; Fax: +33-1-69-15-62-58; e-mail: biarrott@ipno.in2p3.fr.

${ }^{\dagger}$ EC Contract $\mathrm{N}^{\circ}$ FIKW-CT-2001-00179.

${ }^{\ddagger}$ EC Contract N ${ }^{\circ}$ FI6W-516520.
} 


\section{Introduction}

It is now well known that human activity is releasing greenhouse gases at a rate that would cause, sometime in this century, an irreversible global warming effect. Nuclear power, thanks to its negligible contribution to this effect, is more and more advocated worldwide as a promising solution to sustain safely the growing demand for energy. But it is also heavily debated, especially because of the long-term environmental burden from the present generation light-water reactors. Therefore, transmutation of their long-lived radioactive waste has become, more than ever, of high interest. For dedicated transmutation systems, critical reactors loaded with fuel containing large amounts of minor actinides pose safety problems caused by unfavorable reactivity coefficients and small delayed neutron fractions. A sub-critical system using externally provided additional neutrons is very attractive: it allows maximum transmutation rate while operating in a safe manner. An ADS (Accelerator Driven System), coupling a proton accelerator, a spallation target and a sub-critical core, could be used as such a reactor.

A few years ago, a Technical Working Group (TWG) was created by the advisors to several European Research Ministers in order to report on ADS technology. The TWG concluded by presenting in 2001 a "European Roadmap" towards ADS technology [1], proposing the construction of an experimental facility, "XADS", for the 2015 horizon. Triggered by an initiative of the TWG members, the project PDS-XADS, Preliminary Design Study of an eXperimental ADS, was funded by the European Commission in 2002. Performed by 25 participating organizations, PDS-XADS [2,4] contained 5 Working Packages (WP), which studied 3 versions of an XADS: both a molten-metal (eutectic $\mathrm{Pb}-\mathrm{Bi}$ ) and a gas cooled ADS of $100 \mathrm{MW}_{\text {th }}$ class, and a smallerscale $\left(\approx 50 \mathrm{MW}_{\text {th }}\right)$ system based on the MYRRHA project of SCK Mol (Belgium) [6]. WP1, "Global coherence", assured the overall approach to the project, WP2 concerned the safety of these hybrid nuclear systems, WP3 elaborated the accelerator, WP4 the design of core and target, and WP5 the system integration. The PDS-XADS contract has reached its end in October 2004. Below we first give a short summary of the main results as far as the accelerator is concerned, and then present the required $R \& D$, part of the FP6 project EUROTRANS that started in April 2005.

\section{Main specifications for the XADS accelerator}

The main specifications for the XADS accelerator are summarized in Table 1. Evidently, this machine belongs to the category of "HPPA" (high-power proton accelerators). HPPA are presently very actively studied (or even under construction) for a rather broad use in fundamental or applied science $[3,7]$. Compared to other HPPA, many specifications are similar, but it is to be noted that the reliability requirement, i.e. the number of unwanted "beamtrips", is rather specific to ADS. The WP3 studies had to integrate this stringent requirement from the very beginning, since this issue could be a potential "show-stopper" for ADS technology in general.

\subsection{Beam energy}

In the TWG report, an energy of about $1 \mathrm{GeV}$ was selected in view of the physics of spallation reactions and the energy deposition in the target. Yet, since the mission of the $\left(100 \mathrm{MW}_{\text {th }}\right)$ XADS plant is a global demonstration of the operation and safety, but not of industrial waste transmutation, cost considerations favour a lower energy. In any case, a lower limit of about $600 \mathrm{MeV}$ can be set in order to have a reasonable efficiency in neutron production and an affordable beam load on the target window. The XADS accelerator was designed accordingly, but with the requirement that the concept should be upgradeable in energy, and keeping in mind that the smaller-scale (50 $\left.\mathrm{MW}_{\text {th }}\right)$ ADS MYRRHA only requires $350 \mathrm{MeV}$ protons. 
Table 1

PDS-XADS specifications for the proton beam characteristics

\begin{tabular}{ll}
\hline Proton beam parameters & Nominal values \\
\hline Max. beam intensity & $6 \mathrm{~mA} \mathrm{CW}$ on target (10 mA rated) \\
Proton energy & $600 \mathrm{MeV}$ (including $800 \mathrm{MeV}$ upgrade study) \\
Beam entry & Vertically from above \\
Number of Beam Trips & Less than 5 per year (exceeding 1 second) \\
Beam Stability & Energy: $\pm 1 \%$, Intensity: $\pm 2 \%$, Size: $\pm 10 \%$ \\
Beam footprint on target & Gas-cooled XADS: circular $\varnothing 160 \mathrm{~mm}$ \\
& LBE-cooled XADS: rectangular $10 \times 80 \mathrm{~mm}$ \\
& MYRRA: circular, "donut" $\varnothing 72 \mathrm{~mm}$ \\
Intensity modulation & 0.2 ms "interruptions" in CW beam for neutronics measurements, \\
& repetition frequency $0.01-1 \mathrm{~Hz}$ \\
\hline
\end{tabular}

\subsection{Beam intensity and time structure}

The beam intensity can be deduced from the multiplication factor of the sub-critical assembly and the thermal power of the ADS reactor. The PDSXADS studies showed, that for a power level $\mathrm{P}_{\max }$ of $100 \mathrm{MW}_{\text {th }}$, a core load with MOX type fuel and a multiplication factor $\mathrm{k}_{0}=\mathrm{k}_{\max }=0.98$, the proton beam current needed at $600 \mathrm{MeV}$ is about $2 \mathrm{~mA}$ average. Considering a core multiplication factor of $\mathrm{k}_{0}=0.90$ as an extreme lower limit, the current would rise up to about $10 \mathrm{~mA}$. But higher currents have, at least conceptually, also be considered for the XADS accelerator in order to demonstrate the feasibility of industrial operation. Concerning the beam time structure, operation in CW mode was chosen because of its numerous advantages, e.g. lower peak power, vanishing mechanical stress from Lorentz forces in the superconducting cavities, significantly lower $\mathrm{R} \& \mathrm{D}$ effort, and strongly reduced thermo-mechanical stress on the beam window, the target and the subcritical assembly. However, a pulsed operation of the accelerator is possible for time scales that are shorter than the thermal inertia of the different components of the target and the reactor. Such pulsing enables online measurements of the sub-criticality level through dynamic neutron flux analysis [8], that is important in order to ensure a safe operation. Therefore, the XADS accelerator uses $\mathrm{CW}$ operation with the RF continuously applied on the structures, while the beam intensity can be modulated by macro-pulsing.

\subsection{Choice of the basic accelerator concept}

The reliability requirements are essentially related to the number of allowable beam trips, because, frequently repeated, they can significantly damage the reactor structures, the target or the fuel, and also decrease the plant availability. Therefore, beam trips in excess of 1 second duration should not occur more frequently than 5 per year.

Up to now, only sector-focused cyclotrons and linear accelerators ("linacs") are able to provide beam currents in the $\mathrm{mA}$ domain. The $600 \mathrm{MeV}$ cyclotron of PSI [10] delivers about $2 \mathrm{~mA}$ on a routine basis. However, reaching up to $10 \mathrm{~mA}$ is more questionable, and might require two cyclotrons with the beams being funneled together. A given cyclotron also cannot be expanded in energy, so that boosting the energy from 600 to $800 \mathrm{MeV}$, as specified by WP1, would require the full replacement of the final and main stage, being absolutely not cost effective. Furthermore, the industrial transmuter needing about $1 \mathrm{GeV}$, a cyclotron reaches its intrinsic limit because the proton is becoming too relativistic. None of these limitations are present in a linac which can reach $100 \mathrm{~mA}$ intensities without any intrinsic energy limit.

Moreover, the chosen strategy to implement reliability relies on over-design, redundancy and fault-tolerance [11]. This approach requires a highly modular system where the individual components are operated substantially below their performance limit. In contrast to a cyclotron, a superconducting linac, 
with its many repetitive accelerating sections grouped in "cryomodules", conceptually meets this reliability strategy. It further allows keeping the activation of the structures rather low, which is important for radioprotection and hands-on maintenance issues, while even for a well optimized extraction system, the hot spot created in a cyclotron by the lost protons is a serious radiation hazard.

For all these reasons, WP3 concluded that the cyclotron solution for an XADS presents a number of difficulties if not impossibilities - beam trips, doublemachine scheme, funneling, intrinsic current limitation, activation, energy upgrading, pulsing (the requirement to provide pulses for sub-criticality monitoring is a major difficulty for a cyclotron of such power) - that precludes this solution. The reference solution discussed below is therefore a superconducting linac [12]. This assessment is corroborated by the one of OECD/NEA [13]: "Cyclotrons of the PSI type should be considered as the natural and cost-effective choice for preliminary low power experiments, where availability and reliability requirements are less stringent. $C W$ linear accelerators must be chosen for demonstrators and full-scale plants, because of their potentiality, once properly designed, in term of availability, reliability and power upgrading capability".

\section{The XADS reference accelerator}

The proposed reference design for the XADS accelerator, optimized for reliability, is shown in Figure 1, and discussed below.

\subsection{The linac}

For the injector, an ECR source with a normal conducting RFQ is used, followed by warm IH-DTL or/and superconducting CH-DTL structures up to a transition energy still to be optimized. Then a fully modular superconducting (SC) linac accelerates the beam up to the final energy [14].

Below the transition energy, fault-tolerance is guaranteed by means of a "hot stand-by" spare. Above this energy, spoke and, from $100 \mathrm{MeV}$ on, elliptical cavities are used. This superconducting section, that uses highly "de-rated" and independently-powered accelerating components, should be intrinsically "fault-tolerant": beam dynamic calculations showed that an individual cavity failure can be handled at all stages without loss of the beam [15]. Moreover, the performance of the first prototypical cavities has been measured to exceed the specifications for the XADS by a very comfortable over-design safety margin [14,16], that should ensure the required "over-design" criteria.

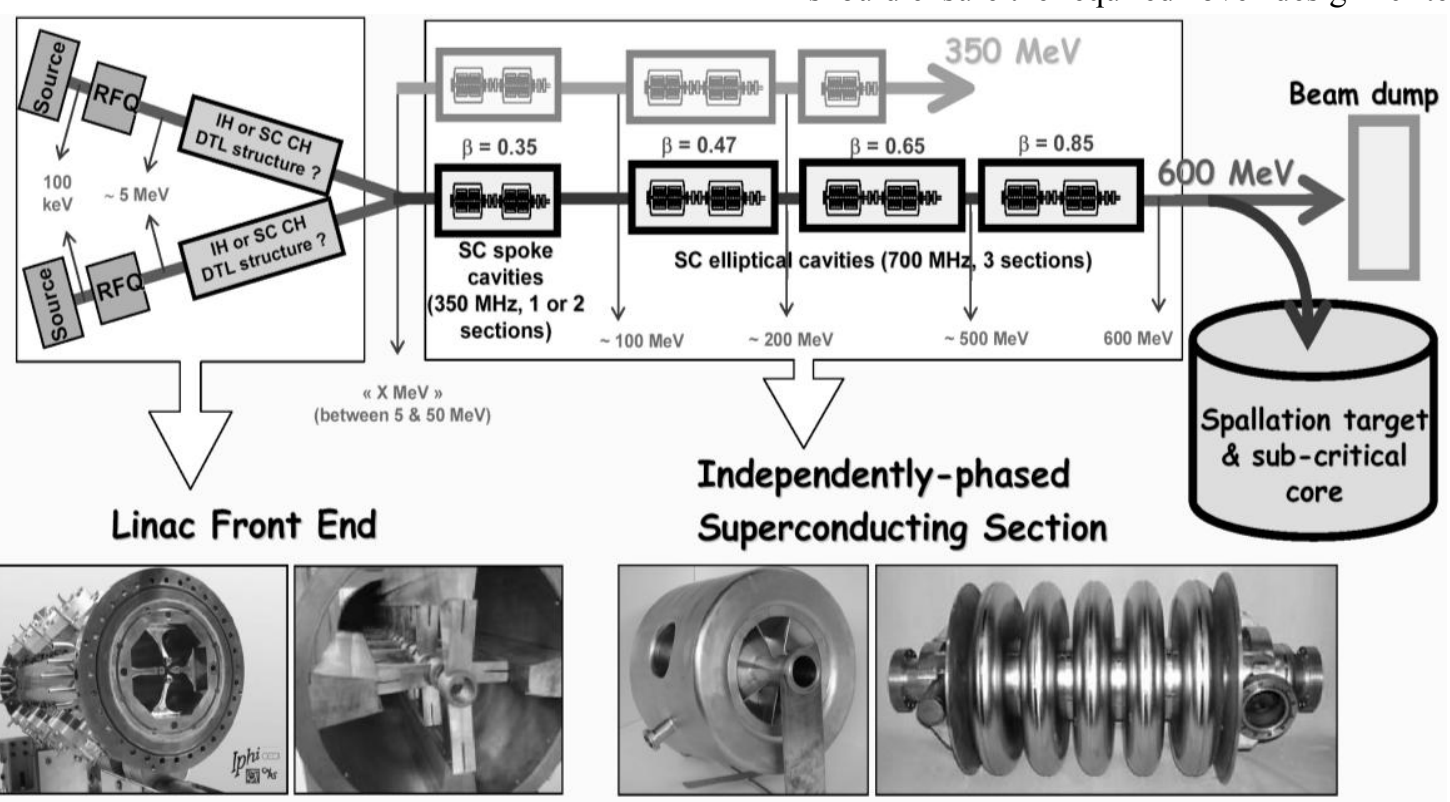

Fig. 1. XADS reference accelerator layout: a doubled injector accelerator is followed by a fully modular spoke and elliptical cavity SC linac. Photos of typical cavity prototypes are shown in the lower part. From left to right: RFQ, CH structure, Spoke, Elliptical 5-cell. 
Another remarkable feature of the concept is its validity for a very different output energy range: 9 cryomodules of $\beta=0.65$ cavities are needed for $350 \mathrm{MeV}$; for $600 \mathrm{MeV}$, simply 10 more cryomodules have to be added ( 7 with $\beta=0.65$ and 3 with $\beta=0.85)$ and 12 additional $(\beta=0.85)$ boost the energy to $1 \mathrm{GeV}$. Therefore, already the small-scale XADS accelerator is fully demonstrative not only of the $600 \mathrm{MeV}$ XADS (and could be converted to it), but even for an industrial machine.

\subsection{The beam transport line}

The objective of the transport line is to safely inject the proton beam onto the spallation target with the specified footprint. A doubly achromatic module composed of two 45 degrees bending dipoles and three focusing quadrupoles has been designed. With scaled magnetic rigidity, the same layout can be used at $350 \mathrm{MeV}$ or $1 \mathrm{GeV}$. Overall, such a beam line is non-dispersive. Thus, the beam position at the target is independent on energy variations, and the beam size independent on the energy spread. Thus, spread and central energy fluctuations from the accelerator will have no effect at the target. The system is however dispersive in the region situated between the two dipoles. Position and size monitors located in this region will be able to provide information on proton energy variations, and to trigger a feedback system.

The footprint is obtained by raster scanning. The pencil-like beam is deflected by fast steering magnets operated at frequencies of 50 to a few hundreds Hertz, and acting in the two transverse directions. Various shapes and various particle distributions are achievable. Four raster magnets will be operated synchronously and independently so that the beam will be always moved at the target if one magnet fails. Redundant fault detection circuits will monitor the magnets current and the magnetic fields so as to ensure proper operation and shut down the beam in case of necessity. Similar systems are used in cancer therapy where they meet stringent requirements.

\subsection{Maintenance strategy and radiation protection}

Proper maintenance has to guarantee that the overdesign margin does not deteriorate and that equipment redundancy is regularly restored if partial failures have occurred. The supervising control system regularly must undergo a complete performance check to ensure that it can replace components-at-fault by readjusting the operational parameters of the overall machine. This requests the development of an expert system, able, while the accelerator is running and delivering nominal beam, to precisely identify and locate equipment that has started to loose rated performance, and/or that is outof-order and to be replaced or repaired. This system provides the database for the scheduled maintenance periods of repair and/or replacement of deteriorated or faulty equipment. This is also in-line with the ALARA (As Low As Reasonably Achievable) principle for the concerned personnel. Indeed, many conditions for the ALARA principle, like enough working space and quick disconnection of sub equipments are actually very much the same as asked by an optimization of the reliability.

The shielding calculations for the XADS accelerator had to be in line with the radiation protection philosophy [17] adopted by the European decree Euratom/96/29. Thus, the goal of the shielding design was to guarantee that, under normal operational conditions, the added integrated dose to personnel will be extremely low, i.e. smaller than the natural background of $1 \mathrm{mS} / \mathrm{y}$. To obtain this goal, the calculations were made using conservative (i.e. pessimistic) assumptions, and assuming an "occupancy factor" of 1, that means a 2000 hoursper-year presence just behind the shield wall where maximum dose rates exist. The design dose rate was $0.5 \mu \mathrm{Sv} / \mathrm{h}$. Some more details are given in [18].

\section{4. $R \& D$ program and roadmap}

The broad field of applications covered by HPPA accelerators explains the remarkable $R \& D$ effort presently underway world-wide. The study, design and testing of the main components of these new generation linear accelerators have contributed to a good synergy by developing complementary activities between many laboratories. The XADS accelerator can profit from this general background and even build on it quite directly. However, a dedicated $R \& D$ programme is needed for the requirement of an extremely low number of beam 
trips. In this spirit, the participants to WP3 have elaborated such a program, focused on reliability and fault tolerance design. It is included the 4 year $6^{\text {th }} \mathrm{FP}$ project EUROTRANS, that started in April 2005.

\subsection{Injector and Intermediate Energy Section}

Concerning the injector section, a thorough campaign to test the reliability of every component of the injector, operated over a long period of time (e.g. a continuous run of many weeks) will be performed "full-scale" with IPHI. Presently under construction in France [19], IPHI, consisting of an already operational ECR source and a $6 \mathrm{~m}$ long RFQ, will deliver, in 2006, its $3 \mathrm{MeV}$ high intensity beams (10 $100 \mathrm{~mA}$ ).

Some basic R\&D is required for the subsequent sections, up to $100 \mathrm{MeV}$, in order to assess a solution simultaneously reliable and economical. While SC components should in principle be deployed from the lowest possible energies onwards, room-temperature structures have nevertheless to be studied and prototyped: the transition energy to the superconducting structures might be higher than the RFQ output and, while room-temperature structures have large RF losses, their development risk is low (well established technology). The superconducting resonators considered here are short and modular in view of the reliability strategy. First SC cavity prototypes are presently successfully tested [20]. It is therefore important to push these developments for spoke- and $\mathrm{CH}-$ structures, by adding helium tanks and power couplers. The final aim of all these developments is to assess the best technical option for the intermediate section of the XADS accelerator based on established demonstrated performance. It might well be a combination of several technologies.

\subsection{High-Energy Section and RF System}

While the R\&D on $704 \mathrm{MHz}$ superconducting elliptical cavities is well advanced in Europe $[7,14,16]$, the demonstration of the full technology is not yet accomplished. Besides the development of the bare superconducting cavity, it is important to prototype each auxiliary system needed for the cavity operation in a real environment (power coupler, RF source, power supply, RF control system, cryogenic system, cryostat...). The construction of a full-scale module with a given beta value (e.g. $\beta=0.5)$ can be considered as a rather general proof-of-principle of the technology, since the higher beta modules are very similar. Moreover, tests with RF at nominal power (although without beam), could be done and used for specific studies, like the RF control system procedure in case of a cavity failure. Indeed, the reaction speed for retuning the whole accelerator, to nominal beam conditions must be less than 1 second for fault-tolerance. Digital techniques are necessary to meet the speed and software configuration requirements.

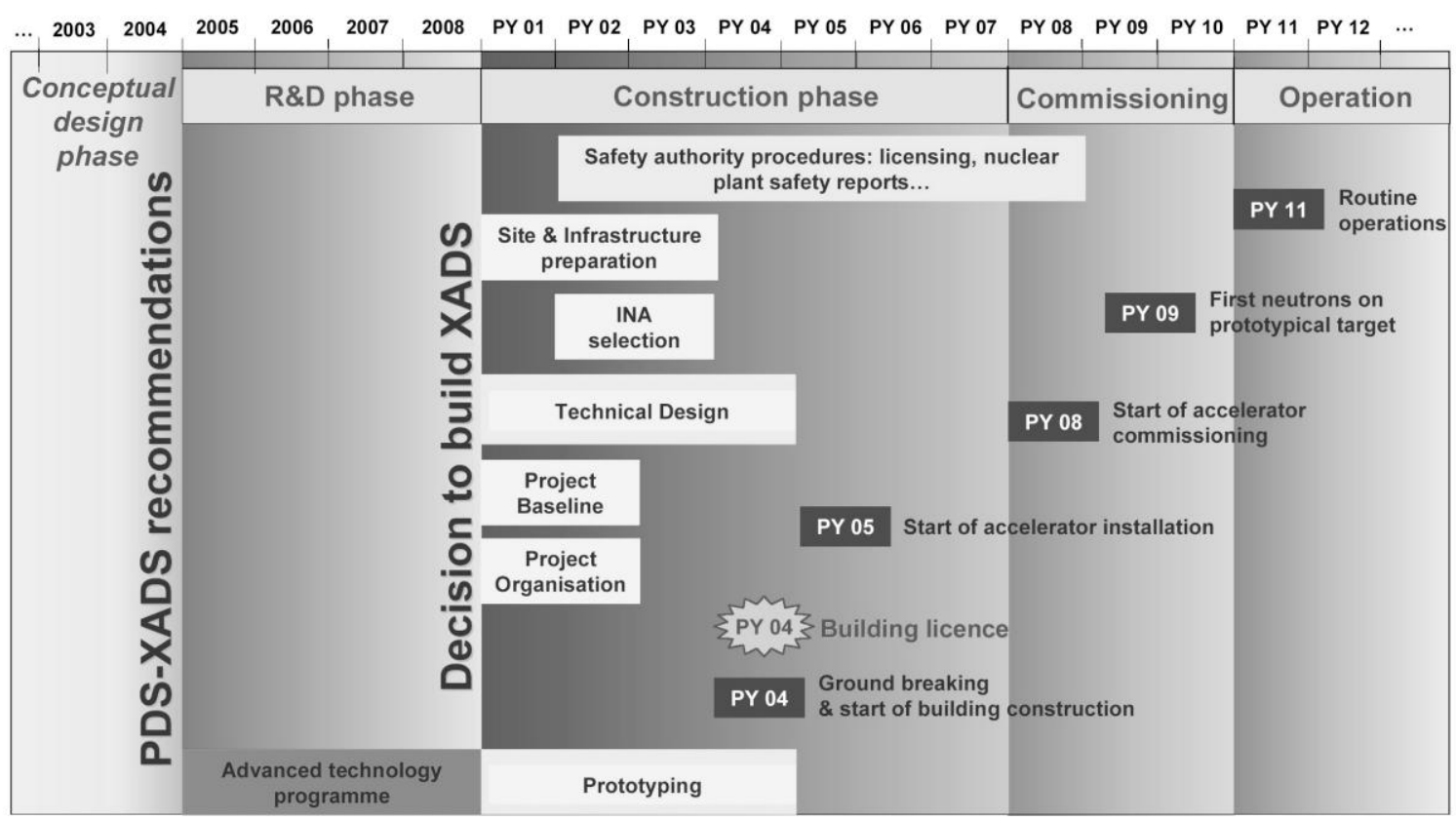

Fig. 2. The roadmap for the accelerator for an experimental ADS demonstrator. 


\subsection{Possible roadmap}

The roadmap for the accelerator is given in figure 2. Consecutive to the advanced technology R\&D phase, the construction of the accelerator of the XADS facility typically may take 7 years. This estimate was based on industrial studies and experience gained from the construction of similar facilities like SNS or ESS [21]. Concerning the cost, prior to routine operation, about $300 \mathrm{M} €$ have been estimated for the $600 \mathrm{MeV}$ machine, including inhouse and external man-power as well as infrastructure investments of $30 \mathrm{M} €$. As far as operation costs are concerned, using superconducting technology from a very low energy range onwards is the most cost effective concept. It is hoped that the R\&D program within EUROTRANS will not only provide answers related to the accelerator performance in the critical area of reliability/availability, but may even allow to slacken some of the presently rather conservative specifications, with positive impact on the price.

\section{Concluding Remarks}

Within the 3 year contract PDS-XADS, a generic and robust technical solution for the XADS accelerator has been developed. A superconducting linac, with its associated doubly-achromatic beam line, has been found to constitute an optimal technical solution. It is also representative for an industrial machine. It is reliable through the rigorous implementation of a highly modular system with derated components operated in a fault-tolerant way. The required $R \& D$ is identified, and a technological validation programme, focused on reliability will be performed within the FP6 project EUROTRANS.

\section{Acknowledgments}

The authors acknowledge the work of all their colleagues of the PDS-XADS WP3 collaboration (from Ansaldo, CEA, CNRS, ENEA, Framatome, IBA, INFN, ITN and University of Frankfurt) on which this short summary paper is based.

\section{References}

[1] Rubbia, C. et al., "A European Roadmap for Developing Accelerator Driven Systems (ADS) for Nuclear Waste Incineration", ENEA, Rome (2001), ISBN 88-8286-008-6.

[2] Abderrahim, H.A., Carluec, B. and Mueller, A.C., "The European Programme", in ref. [3], $3^{\text {rd }}$ meeting.

[3] OECD Nuclear Energy Agency, International Workshops on Utilization and Reliability of High Power Proton Accelerators (HPPA): $1^{\text {st }}$ Meeting, October 13-15, 1998, Mito, Japan, ISBN 92-64-17068-5; $2^{\text {nd }}$ Meeting, November, 22-24, 1999, Aix-enProvence, France, ISBN 92-64-18749; $3^{\text {rd }}$ Meeting, May, $12-$ 16 , 2002, Santa Fe, NM, USA, ISBN 92-64-10211-6; $4^{\text {th }}$ Meeting, May,16-19, 2004, Daejon, Rep. of Korea, in press.

[4] Giraud, B., "Principle Concepts of the XADS Designs in the $5^{\text {th }}$ FP", in ref. [5].

[5] Proceedings of ICRS 10/RPS2004, May 2004, Funchal, Madeira Island, Portugal.

[6] See e.g. Proceedings of the Topical Day from MYRRHA towards XT-ADS, a European Experimental ADS at Mol, November 23, 2004, SCK Mol, Belgium.

[7] Proceedings of the International Workshop on Physics with a multi-MW proton source, CERN, Geneva, 25-27 May, 2004.

[8] Rimpault, C. et al., "Core instrumentation and reactivity Control of the experimental ADS", in ref.[9].

[9] Proceedings of the International Workshop on P\&T and ADS Development, October 6-8, 2003, SCK Mol, Belgium.

[10] Stammbach, Th. et al., Nucl. Inst. Meth. B113 1 (1996).

[11] Pierini, P., "ADS Reliability Activities in Europe", in ref. [3], $4^{\text {th }}$ meeting.

[12] Safa, H., "Design of a XADS linear accelerator", in ref [9].

[13] "Summary of Working Group Discussion on Accelerators", in ref. [3], $3^{\text {rd }}$ meeting.

[14] Biarrotte, J-L., "High Power CW Superconducting linacs for EURISOL and XADS", Proc. of the LINAC2004 Conf., August 16-20, 2004, Lübeck, Germany.

[15] Biarrotte, J-L. et al., "Beam dynamics studies for the fault tolerance assessment of the PDS-XADS linac design", in ref. $[3], 4^{\text {th }}$ meeting.

[16] Junquera, T., "Status and Perspectives of the R\&D programs for the XADS accelerator", in ref. [9].

[17] "Recommendations of the International Commission on Radiological Protection", ICRP Publication 60. Annals of the ICRP 21(1-3) Pergamon Press, Oxford, 1991.

[18] Mueller, A.C., "The PDS-XADS Reference Accelerator and its radioprotection issues", in ref. [6].

[19] Beauvais, P.Y., "Recent Evolutions in the Design of the French High Intensity Proton Injector (IPHI)", Proc. of the EPAC 2004 Conf., July 5-9, 2004, Luzern, Switzerland.

[20] Olry, G. et al., "Recent developments on superconducting beta 0.35 and beta 0.15 spoke cavities at IPN for low and medium energy sections of proton linear accelerators", Proc. of the EPAC 2004 Conf., July 5-9, 2004, Luzern, Switzerland.

[21] See e.g. "The ESS project Volume III update - Technical report", June 2003. 